\title{
New Polaritonic Materials in the THz Range made of Directionally Solidified Halide eutectics
}

\author{
R. I. Merino, M.F. Acosta and V. M. Orera \\ Instituto de Ciencia de Materiales de Aragón, CSIC-Universidad de Zaragoza, \\ Pedro Cerbuna 12, E50009-Zaragoza, Spain.
}

Corresponding author:

R. I. Merino

Instituto de Ciencia de Materiales de Aragón, CSIC-Universidad de Zaragoza, Pedro Cerbuna 12, E50009-Zaragoza, Spain

Tel: +34876553447

r.merino@csic.es

Accepted J. Europ. Ceram. Soc. $20^{\text {th }}$ October 2013

Keywords: (up to 5) THz, Metamaterials, Eutectics, Alkali halides, Polaritonics

\begin{abstract}
Directionally solidified alkali halide binary eutectics have been recently proposed as $\mathrm{THz}$ polaritonic metamaterials based on their ordered microstructure and the suitable phonon-polariton resonances in the $\mathrm{THz}$ range of the spectrum. In the present work we focus on the search of new available eutectic systems both binary and ternary eutectics with well-ordered fibrous or lamellar microstructures and interparticle distances from 1 to several tens of microns. Simple effective homogenization models have been used to calculate effective permittivity and transmittance in the $\mathrm{THz}$ range of eutectic slices. This lets us identify the electromagnetic spectral ranges where hyperbolic dispersion is expected together with a significant transmittance value. The hyperbolic dispersion range shifts with microstructure size, that is, with growth parameters, showing that the materials response can be finely tuned by the manufacture conditions. Applications with these materials cover the electromagnetic range from 5 to $20 \mathrm{THz}$.
\end{abstract}




\section{INTRODUCTION}

$\mathrm{THz}$ waves are used in communications, imaging or sensing technologies. Nowadays also, there are new laser sources that give $\mathrm{THz}$ technology increased importance. According to $\mathrm{M}$. Tonouchi [1], higher power $\mathrm{THz}$ sources, more sensitive $\mathrm{THz}$ sensors and more functional materials are still required to realize all kinds of imagined applications.

In particular, there is a need for passive elements that would serve to handle (focussing, shaping and guiding) the $\mathrm{THz}$ beams. Suitable materials for these devices include materials with very large, near zero or negative dielectric constant in the appropriate wavelength range. Small losses are also required so that subwavelength guiding or focusing can be made possible. Existing metamaterials in the $\mathrm{THz}$ range would fulfil these requirements but the fabrication methods based on micro/nano machining of 2D or 3D architectures are yet expensive and time consuming [2]. Large 2D metal-dielectric metamaterials have been produced by drawing techniques, using technologies similar to those developed for photonic crystal optical fibers fabrication [3, 4]. Recently, a bottom-up technique to fabricate composite meta-atoms for photonic wavelengths by self-assembling of metallic particles has also been implemented [5]. Negative effective permeability or permittivity has also been obtained in composite materials consisting of aligned metallic or polaritonic wires embedded in a dielectric matrix $[6,7,8]$. The reason is that frequency ranges of negative permittivity (with hyperbolic dispersion relations) or negative permeability occur in some uniaxially anisotropic materials. These anisotropic media may present lower losses than resonance based negative permeability, negative permittivity materials.

Directionally solidified eutectics (DSEs) are in-situ self-organized composites of two or more crystalline phases. The use of this kind of composite to manufacture metamaterials has been proposed previously $[9,10]$. The microstructure of DSEs may consist of rods of one phase embedded into the matrix of the other phase or of alternate lamellae with a dominant alignment direction (the pulling direction or the growth direction) [11]. DSEs mimic in bulk form anisotropic phase orderings that are otherwise achieved by costly top-down or nano-fabrication approaches [12]. The characteristic length of the transverse microstructure of DSEs for well ordered, coupled microstructures spans from tens of nanometers to tens of microns. These are 
subwavelength dimensions for $\mathrm{THz}$ radiation, and thus, appropriate homogenization theories can be applied to approximately describe the propagation of $\mathrm{THz}$ waves in these materials. Required low losses and large dielectric contrast are achieved by alkali halides in the THz range (or FIR). They show wide ranges of transparency in this region and the negative or very large dielectric constant is provided by the phonon-polariton resonance which, moreover, in these compounds, consists of a single mode. In addition, having small values of the entropy of melting, coupled, halide eutectic composites generate well-ordered microstructures presenting anisotropic dielectric behaviour [13].

Recently, polaritonic metamaterial behaviour has been proved in $\mathrm{NaCl}-\mathrm{LiF}$ and $\mathrm{KCl}-$ LiF rod-like directionally solidified eutectics [14]. They were solidified by the Bridgman method so that $14 \mathrm{~mm}$ diameter ingots were prepared and characterized by FTIR reflectance spectroscopy. The IR reflectance of $\mathrm{KCl}-\mathrm{LiF}$ was reasonably well reproduced by electromagnetic calculations assuming a perfectly hexagonal arrangement of LiF rods inside the chloride matrix. The response contained the Mieresonance of the $\mathrm{LiF}$ polaritonic rods at large rod diameters and converged to a rod-size independent LiF polaritonic resonance at small rod-size diameters. In the latter case, a simple Maxwell-Garnett effective medium model can describe the reflectance spectra. This description, shown by S. Foteinopoulou [15] to be suitable whenever the ratio or periodicity (a) to wavelength in vacuum $(\lambda)$ satisfies the relationship $a / \lambda<0.1$, generates a material with hyperbolic dielectric permittivity in certain wavelength ranges that would allow the subwavelegth guiding and large light transmittance in Fabry-Perot cut slices $[16,17,18]$. The potentiality of those self-organized easy-to-grow materials has been, consequently, put forward, and the search for new eutectic systems working at different wavelengths as well as the investigation of real materials with its intrinsic variability in size of the dispersoids and experimental demonstration of the predicted properties requires more work.

In the present manuscript we report the microstructure and $\mathrm{THz}$ dielectric permittivity in the quasistatic approximation (sufficiently long wavelength limit) of some selected alkali halide eutectics. We aim at showing the potentiality of these materials as a source for metamaterials or polaritonic photonic crystals in the $\mathrm{THz}$, and at encouraging theoretical as well as experimental work in understanding and 
identifying properties and applications hidden in those easy-to-manufacture selfassembling composites.

\section{BACKGROUND}

As we have said in the Introduction, the materials chosen for this study are the simplest halide salts. The $\mathrm{THz}$ properties of alkali halides are determined by the phonon-polariton interaction. In the simple rock-salt structure the simplest description contains one single phonon polariton with transverse $\left(\omega_{\mathrm{T}}\right)$ and longitudinal $\left(\omega_{\mathrm{L}}\right)$ frequencies so that the dielectric constant (real part) is negative in the interval $\omega_{\mathrm{T}}<\omega<$ $\omega_{\mathrm{L}}$ (Reststrahlen region), and positive outside this interval. Damping of the phonon polariton broadens the $\operatorname{Im}(\varepsilon)$ curve, and $\varepsilon$ does not diverge at $\omega=\omega_{\mathrm{T}}$. In figure 1 we sketch the frequency range of negative permittivity, $\operatorname{Re}(\varepsilon)<0\left(\omega_{\mathrm{T}}\right.$ and $\omega_{\mathrm{L}}$ values are the extreme points of the segments on the plot) for several materials (alkali halides, fluorite and $\mathrm{MgO})$. The static $\left(\omega<<\omega_{\mathrm{T}}\right)$ and high frequency $\left(\omega>>\omega_{\mathrm{L}}\right)$ approximate values of $\operatorname{Re}(\varepsilon)$ are also given in the diagram. For the chosen materials it can be seen that $\omega_{\mathrm{T}}$ ranges between $142 \mathrm{~cm}^{-1}$ (4.26 THz) for $\mathrm{KCl}$ to $306 \mathrm{~cm}^{-1}(9.17 \mathrm{THz})$ for $\mathrm{LiF}$ or $401 \mathrm{~cm}^{-}$ ${ }^{1}(12.0 \mathrm{THz})$ for $\mathrm{MgO}$; while $\omega_{\mathrm{L}}$ ranges from $214 \mathrm{~cm}^{-1}(6.41 \mathrm{THz})$ for $\mathrm{KCl}$ to $659 \mathrm{~cm}^{-1}$ (19.8 THz) for $\mathrm{LiF}$ and $718 \mathrm{~cm}^{-1}(21.5 \mathrm{THz})$ for $\mathrm{MgO}$ [19].

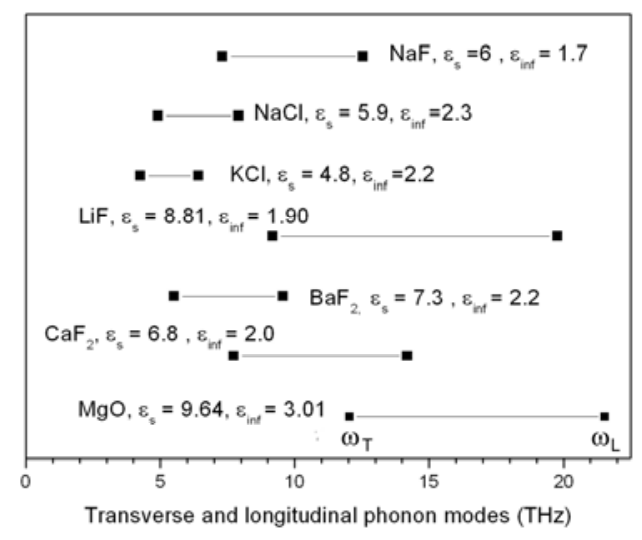

Figure 1: Reststrahlen region and permittivity of some halides and $\mathrm{MgO}$ below $\left(\varepsilon_{\mathrm{s}}\right.$, static permittivity) and above ( $\varepsilon_{\text {inf }}$, high frequency permittivity) the phonon-polariton dispersion region. 
The materials used in this study have been prepared by solidification from the melt of eutectic mixtures [11]. Directional solidification of eutectic composites consisting of phases with low melting entropy produces a solid with a microstructure of separated phases, lamellae, fibres or more complex morphologies, well aligned along the solidification direction. Unidirectional alignment along the solidification direction induces anisotropic properties in otherwise isotropic composites. The size and morphology of the eutectic structure can be modified by simply changing the growth rate according to the following relationship between interphase spacing $a$ and the solidification rate $\mathrm{v}$ :

$$
a^{2} \cdot v=K_{1}
$$

$K_{1}$ is a constant which depends on the eutectic system under study. Hence, finer microstructures would be obtained at higher growth rates. The eutectics utilized in this study are composed of low melting entropy single phases and consequently, solidify on a coupled growth regime with well aligned, highly homogeneous microstructures. For volume fractions of the minority phase $f<0.3$ we obtain fibrous microstructures. Otherwise the expected microstructure is lamellar.

With respect to the electromagnetic properties, the permittivity of an anisotropic composite formed by phase $f$ embedded in phase $m$ (at volumetric fraction 1-f), assuming that the microstructure consists of long fibers or platelets of phase $f$ aligned in the $\mathrm{Z}$ direction and with deep subwavelength size in the $\mathrm{XY}$ transverse plane, can be calculated using an arithmetic average for parallel polarization (equation 2a) or the Maxwell formula for perpendicular polarization (eq. 2b). The effective permittivity values are [20]:

$$
\begin{array}{ll}
\text { For } \mathrm{E} / / \mathrm{z} \text { axis (fiber long axis) } & \varepsilon_{\|}=f \varepsilon_{f}+(1-f) \varepsilon_{m} \\
\text { For } \mathrm{E} \perp \mathrm{z} \text { axis (fiber long axis) } & \varepsilon_{\perp}=\varepsilon_{m} \frac{(1+f) \varepsilon_{f}+(1-f) \varepsilon_{m}}{(1-f) \varepsilon_{f}+(1+f) \varepsilon_{m}}
\end{array}
$$

Equations $2 \mathrm{a}$ and $2 \mathrm{~b}$, will be used in the following to predict the electrical response of the fibrous composites presently subject of study, as a first approximation. $\varepsilon_{f}$ and $\varepsilon_{m}$ denote the permittivity of phase $f$ (fibers or platelets) and phase $m$ (matrix), respectively. Note that this simple description does not incorporate any information on the size or shape of the dispersoids inside the matrix. It only takes into account the fact that the 
composite is a $2 \mathrm{D}$ structure, infinite in the $\mathrm{Z}$ direction. If $\mathrm{E} / /$ rods, equation $2 \mathrm{a}$, resonances appear at the optical transverse polaritonic modes, $\omega_{\mathrm{TO}}$, of component phases. However, the poles of equation $2 \mathrm{~b}$ correspond to resonant modes localized at the interface between rods and matrix [2].

Also notice that the resonances occurring for $\mathrm{E} \perp \mathrm{z}$ move when the volume fraction of the minority phase changes. In fact for low volume fractions, when $f$ is very small, the surface resonances excited with E perpendicular to rods take place at $\varepsilon_{\mathrm{f}}=-\varepsilon_{\mathrm{m}}$, that is, at a frequency, $\omega_{\text {sf }}$, lower than the optical longitudinal mode, $\omega_{\mathrm{LO}}$, of the fibers, and at $\omega_{\mathrm{sm}}$ below and near to $\omega_{\mathrm{LO}}$ of the matrix, as long as the reststrahlen regions of matrix and fibers are sufficiently apart from one another. In directionally solidified eutectics, the maximum filling factor for a fibrous microstructure is approximately $1 / 3$. If $f=1 / 3$, the resonances appear at $\varepsilon_{\mathrm{f}}=-2 \varepsilon_{\mathrm{m}}$. So, $\omega_{\mathrm{sf}}$ decreases and moves away from $\omega_{\mathrm{LO}}$ of the fiber and $\omega_{\mathrm{sm}}$ moves in the opposite direction, coming nearer to $\omega_{\mathrm{LO}}$ of the matrix. As we see, the frequencies of the composite polaritonic resonances may be tuned by changing the volume fractions of the minority phase.

When the microstructure is lamellar, being the $\mathrm{Z}$ axis perpendicular to the lamellae, the appropriate formulas to calculate the effective permittivity, given by arithmetic and harmonic averages, are [20]:

For E // XY (lamellae plane)

$$
\varepsilon_{\|}=(1-f) \varepsilon_{1}+f \varepsilon_{2}
$$

For $\mathrm{E} \perp \mathrm{XY}$ (lamellae plane)

$$
\varepsilon_{\perp}=\left(\frac{1-f}{\varepsilon_{1}}+\frac{f}{\varepsilon_{2}}\right)^{-1}
$$

Where $f$ is now the volume fraction of phase 2 .

Equation $3 \mathrm{~b}$ has also a pole, and so, resonances are also expected for this perpendicular polarization when $\varepsilon_{1} / \varepsilon_{2}=(f-1) / f$. This resonance causes absorption near $\omega_{\text {LO }}$ of the minority phase. It corresponds to longitudinal bulk modes excited inside the thin lamellae that are observed, for example, in thin film absorption experiments 
performed in $\pi$-polarization under non normal incidence [21]. As $f$ increases, this frequency also shifts to lower values.

Apart from the shift, in both cases, an increase in volume fraction of the minority phase, increases the intensity of its associated resonance (surface modes in the fibrous microstructure or longitudinal optical mode resonance in the lamellar case).

It is relevant to recall now that bulk perpendicular longitudinal-optical modes of lamellae shift to frequencies larger than $\omega_{\mathrm{LO}}$ as the thickness of the lamellae increases beyond several microns. The rod surface modes excited in perpendicular polarization at $\omega_{\text {sf }}$ also shift towards lower energies and higher order modes appear for rod diameters larger than several microns. Those features are not predicted by the simple model used here but they should be taken into account as the size of the microstructural features increases beyond several microns.

Using the effective permittivity values given above, the polarized reflectance or the transmittance of bulk samples can be calculated making use of the Fresnel equations. If we write the complex refractive index of the medium, assuming that the permeability is 1 , as

$$
\tilde{n}=n+i \kappa=\sqrt{\varepsilon}
$$

The transmittance for normal incidence of a slice with thickness $d$ in air $\left(n_{\text {air }}=1\right)$ is given by $\mathrm{T}=|\tau|^{2}$, where $\tau$ is the amplitude coefficient given by:

$$
\tau=\frac{t_{12} t_{21} \exp (i \tilde{n} k d)}{1-r_{12} r_{21} \exp (2 i \tilde{n} k d)}
$$

with the Fresnel transmission and reflection amplitude coefficients given by

$$
\begin{aligned}
& t_{12}=\tilde{n} t_{21}=\frac{2}{1+\tilde{n}} \\
& r_{12}=-r_{21}=\frac{\tilde{n}-1}{\tilde{n}+1}
\end{aligned}
$$

These equations will be used in this paper to calculate the transmittance of real systems. 


\section{EXPERIMENTAL METHODS}

Eutectic ingots of $14 \mathrm{~mm}$ diameter have been grown using the directional solidification Brigdman method in a resistive furnace with up to $40 \mathrm{~K} / \mathrm{cm}$ gradient at the solid-liquid interface. The starting powder compositions are the ones of the corresponding eutectics, given in table I. Pulling rates could be varied between $1 \mathrm{~mm} / \mathrm{h}$ and $100 \mathrm{~mm} / \mathrm{h}$. The grown materials were cut with diamond wafering blade and polished with diamond pastes and suspensions, either dry or with wet vehicles, as required by the different solvent sensitivity of the different materials. Optical microscopy and scanning electron microscopy (SEM) was used to observe the samples microstructure. The SEM images were taken in a Merlin Field Emission Scanning Electron Microscope SEM from Carl Zeiss (Germany). Phase interspacing and volumetric fraction of the phases were obtained by image analysis using the software Digital Micrograph from Gatan Inc. Thin slices of the materials were obtained by lapping around $0.5 \mathrm{~mm}$ thick slices until thickness was below $100 \mu \mathrm{m}$. Transmittance measurements with unpolarized light at normal incidence have been made with a Perkin Elmer SPECTRUM 100 FT-IR spectrophotometer.

\section{MICROSTRUCTURE OF DSE HALIDES.}

In table I we record some binary and ternary halide eutectics that have been grown in our laboratory. Typical microstructures are given in figure 2 for binary eutectics and in figure 3 for one ternary eutectic. The binary eutectics with a low volumetric fraction of the dispersed phase show ordered microstructures consisting of rods embedded in matrix (marked with $\mathrm{F}$ in table I, column number 2). When the tendency to faceting of one or both components is stronger ribbons instead of rods, as is the case of $\mathrm{NaF}$ in $\mathrm{NaCl}[22,23]$ or faceted rods, in $\mathrm{MgO}-\mathrm{CaF}_{2}$ are observed [24]. The systems shown in Table I grow coupled so that large ingots of ordered eutectics can be prepared. In figure 4 we give an optical photograph of transverse and longitudinal slices, around $1 \mathrm{~mm}$ thickness, of the eutectic $\mathrm{KCl}-\mathrm{LiF}$. The material is rather transparent in the transverse direction, which is an indication of the good longitudinal alignment of the LiF fibers inside the matrix. Probe of this is that at NIR wavelengths $(3 \mu \mathrm{m})$, in-line transmittance 
in transverse sections of this material is larger than $50 \%$ for samples grown at 6,20 and $50 \mathrm{~mm} / \mathrm{h}$ and $1 \mathrm{~mm}$ thick.
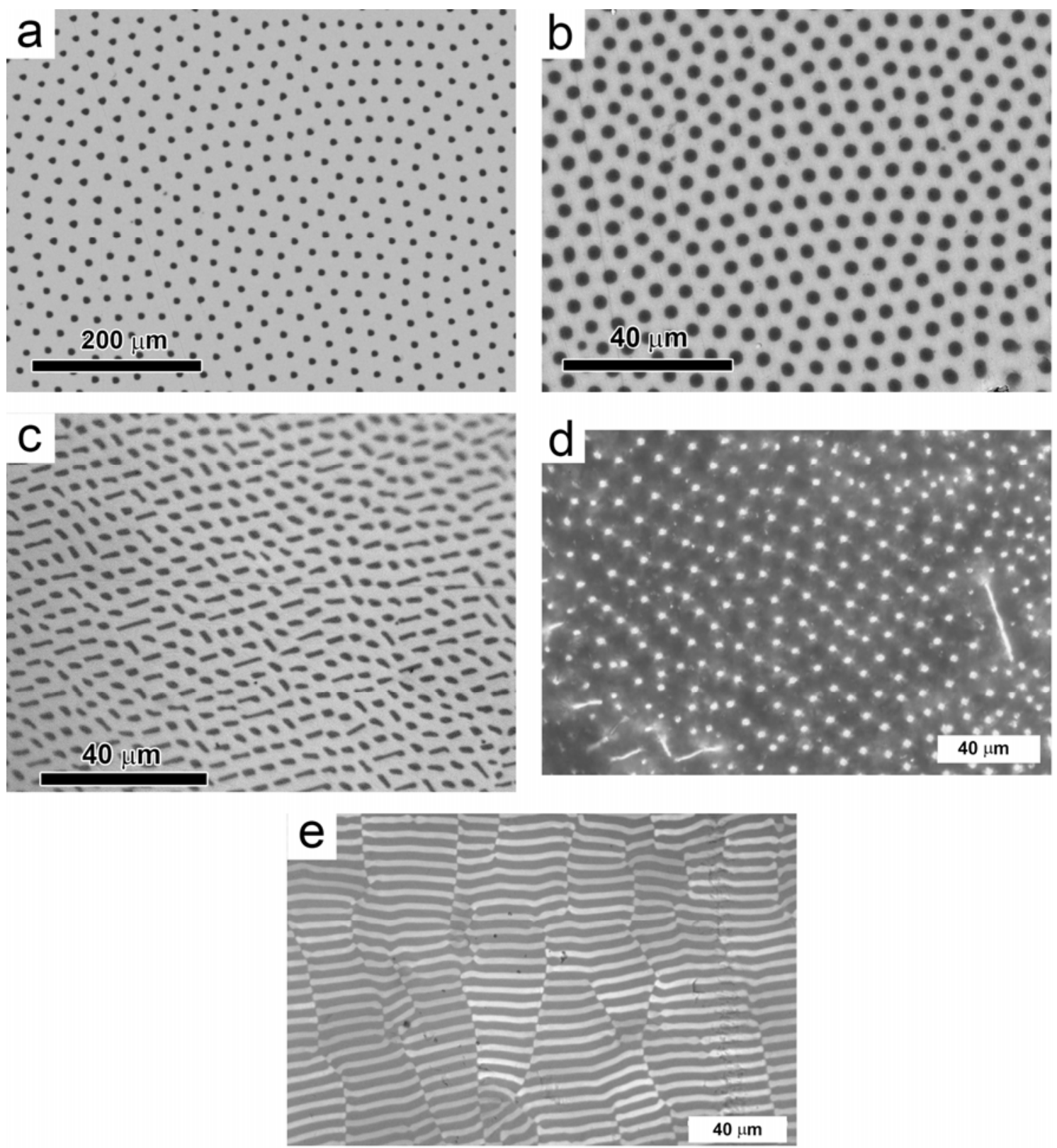

Figure 2: Micrographs of transverse cross-sections of the binary eutectics KCl-LiF (a), NaCl-LiF (b), $\mathrm{NaCl}-\mathrm{NaF}$ (c), $\mathrm{CaF}_{2}-\mathrm{MgO}$ (d) and $\mathrm{CaF}_{2}-\mathrm{LiF}$ (e). a, b and c are SEM micrographs; $d$ and e are transmission optical micrographs. The dark phase is the fluoride phase in figures a to d. Figures $\mathrm{d}$ and e are reproduced with permission from Merino RI, Pena JI, Larrea A, de la Fuente GF and Orera VM. Melt grown composite ceramics obtained by directional solidification: structural and functional applications. Recent Res. Devel. Mat. Sci. 2003; 4: 1-24. 
The average interfiber distances or interlamellar distances, span a range from $1 \mu \mathrm{m}$ to several tens of microns $[22,23,24,25,26,27,28,31]$. Pulling rates from $1 \mathrm{~mm} / \mathrm{h}$ to $100 \mathrm{~mm} / \mathrm{h}$ are achievable in Bridgman method with interphase spacings following equation (1).
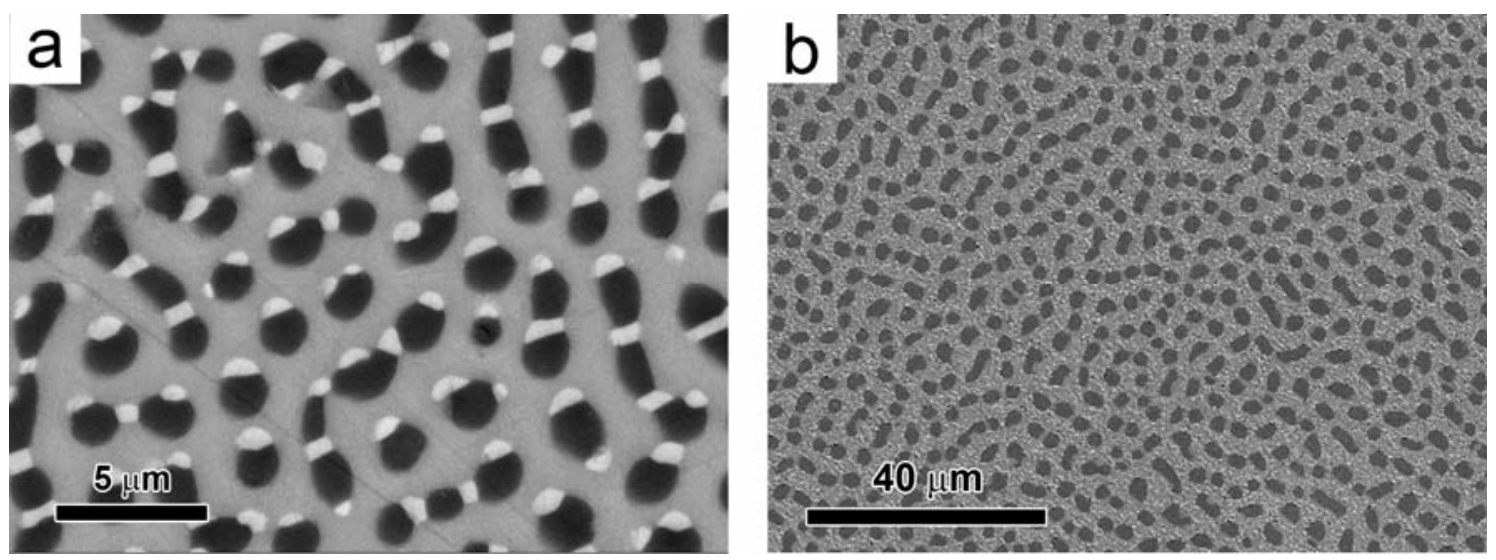

Figure 3: Micrographs of transverse cross-sections of the $\mathrm{NaCl}-\mathrm{LiF}-\mathrm{CaF}_{2}$. The dark phase is $\mathrm{LiF}$. The lighter one corresponds to $\mathrm{CaF}_{2}$. The matrix (grey phase) is $\mathrm{NaCl}$.

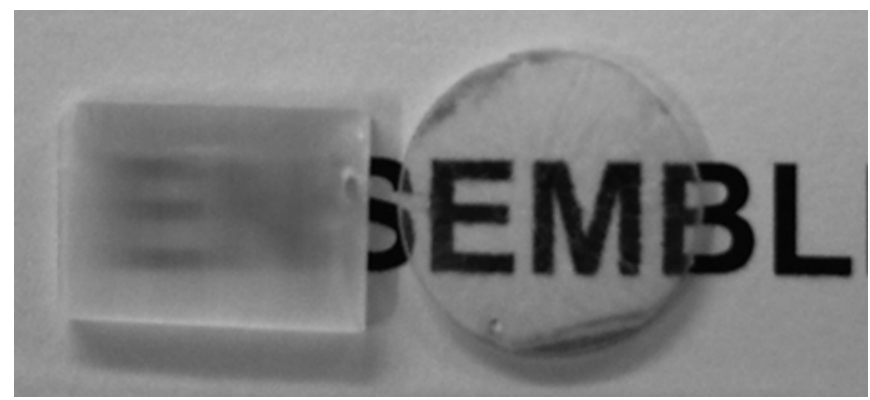

Figure 4: Optical photograph of a longitudinal (left) and transverse (right), polished section of a $\mathrm{KCl}-\mathrm{LiF}$ eutectic grown at $20 \mathrm{~mm} / \mathrm{h}$. The slices are approximately $1 \mathrm{~mm}$ thick, and the diameter of the transverse cut is $13 \mathrm{~mm}$.

For samples with inter-rod distances below approximately $5 \mu \mathrm{m}$, the quasistatic approximation can be applied in the polaritonic region (wavelengths from 15 to $70 \mu \mathrm{m}$ ), with the effective dielectric constant being insensitive to small variations of rod diameters that might result from the directional solidification procedure. Increasing the number of components in eutectic mixtures has been used previously to get finer 
microstructures [29]. Upon this assumption, we have grown the ternary system NaCl$\mathrm{LiF}_{-} \mathrm{CaF}_{2}$. A homogeneous eutectic microstructure has been achieved by Bridgman at pulling rates up to $10 \mathrm{~mm} / \mathrm{h}$. Above this value, with this growth method some ternary colonies form. The microstructure is shown in figure 3, corresponding to $6 \mathrm{vol} \% \mathrm{CaF}_{2}$ +30 vol \% $\mathrm{LiF}$ in a $54 \mathrm{vol} \% \mathrm{NaCl}$ matrix. The minimum interparticle spacing ( $\mathrm{LiF}$ to $\mathrm{LiF}$ centers in transverse cross-section) achieved for pulling rates of $40 \mathrm{~mm} / \mathrm{h}$ amounts to $2.5 \mu \mathrm{m}$. This is to be compared with $5.5 \mu \mathrm{m}$ in $\mathrm{LiF}-\mathrm{NaCl}$ at the same pulling rate [30], or 3.6 $\pm 1 \mu \mathrm{m}$ in $\mathrm{LiF}-\mathrm{KCl}$ [31]. This makes some gain over the binary system as can be seen comparing the sizes in figures $2 b$ and $3 b$, which are crystals grown at the same pulling rate. More importantly, the size of the individual dispersoids (transverse crosssection) is here also smaller, mainly much smaller for the $\mathrm{CaF}_{2}$ minority phase. It is to remark the very homogeneous microstructure that is achieved, consisting of composite with dispersed phases $\mathrm{CaF}_{2}-\mathrm{LiF}$ inside a matrix of $\mathrm{NaCl}$. As this ternary eutectic has 30 vol\% of LiF distributed as dispersed elongated and aligned phase inside a matrix consisting mainly of $\mathrm{NaCl}$, the intensity of the resonant modes in perpendicular polarization also increases. The maximum pulling rate for coupled growth can most probably be shifted to larger pulling rates (and smaller microstructures) using different growth procedures that present larger thermal gradients at the solid-liquid interphase.

\section{THz PROPERTIES OF ALKALI HALIDE EUTECTICS}

Early studies of the $\mathrm{THz}$ properties (IR reflectance measurements) of some alkali halide eutectics were reported for NaF-LiF lamellar eutectics [32]. Authors argued that they observed the resonances excited at the interface of both polaritonic crystals. Recently, A. Reyes-Coronado et al. [14] investigated the far infra-red reflectance in the 3 to $11 \mathrm{THz}$ range of $\mathrm{KCl}-\mathrm{LiF}$ and $\mathrm{NaCl}-\mathrm{LiF}$, for both electric field parallel or perpendicular to $\mathrm{LiF}$ rods polarizations and different rod diameters. The $\mathrm{LiF}$ rod Mie scattering mode that is excited with parallel polarization was observed to shift to longer wavelengths with the increase of the rod diameter, while, at the smaller diameters, the Maxwell-Garnett approximation described to a high degree of precision the material reflectance in this range. Calculations of the mode pattern in these composites followed for waves propagating along $[16,17]$ or across $[17,18]$ the $\mathrm{LiF}$ rods at different wavelengths (10 THz and $14 \mathrm{THz})$. 
The interest in this material relies on the characteristic hyperbolic dispersion that the aligned eutectics present at several regions of the electromagnetic spectrum. In figure 5a we give the calculated permittivities (using equations 2) of the eutectic $\mathrm{NaCl}-\mathrm{LiF}$ using as $\mathrm{NaCl}$ and $\mathrm{LiF}$ dielectric constant the one-mode Lorentz model with the parameters given in the figure caption, between 3 and $20 \mathrm{THz}$. It can be seen that at $5 \mathrm{THz}$ the $\mathrm{NaCl}$ matrix reststrahlen region dominates, with excitation of bulk resonances of the matrix (E// to rods) and perpendicular ( $\mathrm{\perp}$ to rods) polarizations. In parallel polarization, moreover, the bulk resonances of LiF rods appear at 9.22 THz. Finally, around $14 \mathrm{THz}$, another resonance appears in perpendicular polarization. This corresponds to the pole of equation $2 b$, which, at very small diameters and very small volume fraction of fibers, equals to the surface phonon-polariton resonance of LiF cylinders, which is excited only in perpendicular polarization [21]. This resonance shifts with rod diameter and is sensitive to the shape of the dispersoids. It can be thus tailored at will, as well as the bulk LiF-rods Mie-resonance excited in parallel polarization.
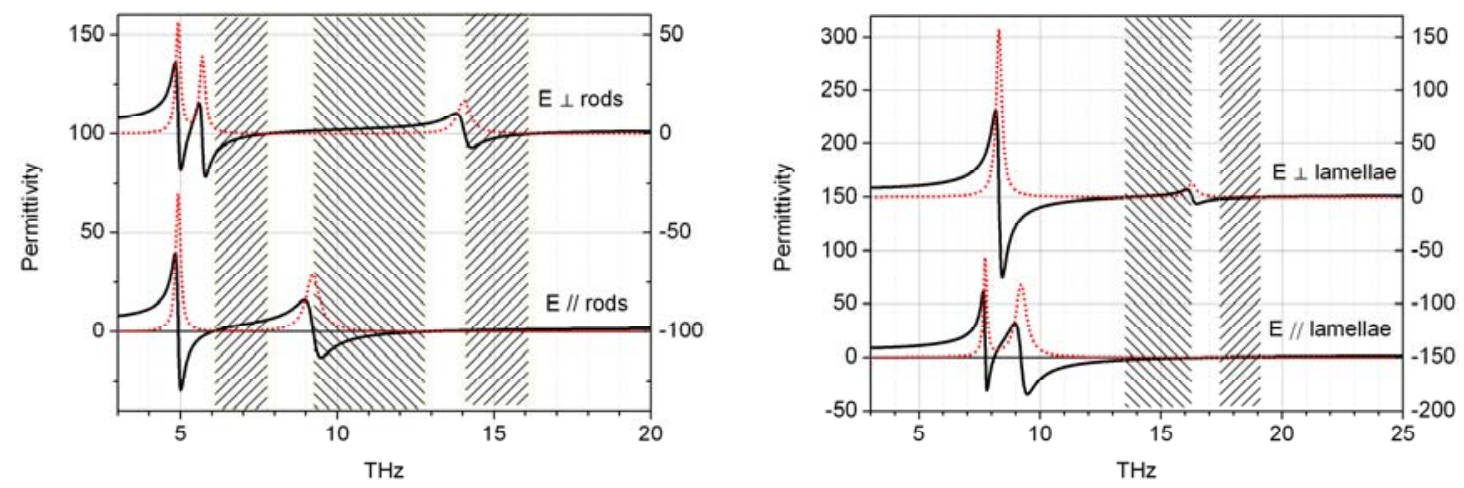

Figure 5: a) Permittivity of the eutectic NaCl-LiF in both polarizations (E parallel to the long direction of the LiF fibers (lower part) and perpendicular (upper plot)). b) Permittivity of the eutectic $\mathrm{CaF}_{2}-\mathrm{LiF}_{\text {in }}$ both polarizations (E parallel to the lamellae (lower part) and perpendicular (upper plot)). Solid line: $\operatorname{Re}(\varepsilon)$, dotted line: $\operatorname{Im}(\varepsilon)$. Fit parameters for the permittivity of the constituents: $\mathrm{NaCl}: \varepsilon_{0}=5.67, \varepsilon_{\infty}=$ 2.303, $v_{\mathrm{TO}}=4.92 \mathrm{THz}, \gamma=0.18 \mathrm{THz}$; LiF: $\varepsilon_{0}=8.705, \varepsilon_{\infty}=2.027, v_{\mathrm{TO}}=9.22 \mathrm{THz}, \gamma=0.527 \mathrm{THz}$. CaF2: $\varepsilon_{0}=6.621, \varepsilon_{\infty}=2.131, v_{\mathrm{TO}}=7.73 \mathrm{THz}, \gamma=0.156 \mathrm{THz}\left(v_{\mathrm{TO}}\right.$ denotes the transverse optical mode frequency and $\gamma$ the damping constant). The regions where the materials have indefinite permittivity are shadowed. 

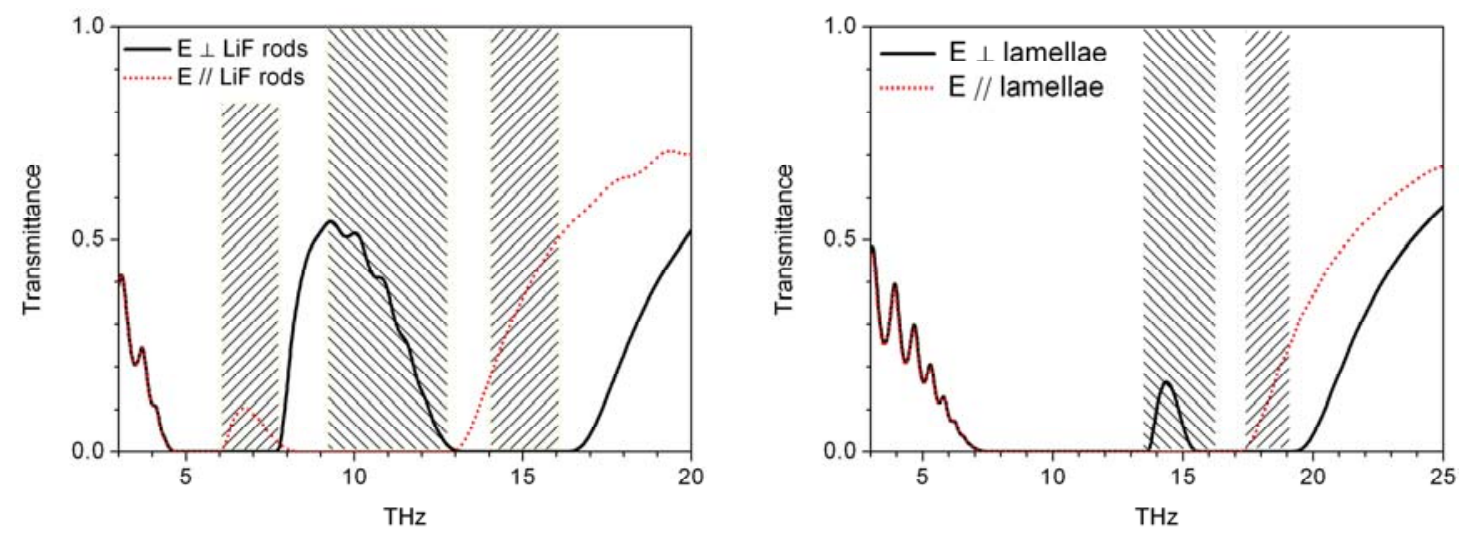

Figure 6: Transmittance of (a) $\mathrm{NaCl}-\mathrm{LiF}$ and (b) $\mathrm{CaF}_{2}-\mathrm{LiF}$ slices, $50 \mu \mathrm{m}$ thick for parallel (dotted line) and perpendicular (solid line) polarizations. Calculations using the data of figure 5. The regions where the materials have indefinite permittivity are shadowed.

The permittivity values given in figure 5a would result in a transmittance for a $50 \mu \mathrm{m}$ thick slab as given in figure 6 a. This shows clearly where we do expect to have enough transmitted intensity. At around $6.7 \mathrm{THz}$, the transmittance in parallel polarization is 10 $\%$, with $\varepsilon^{\prime} / /$ positive (2.8) and $\varepsilon^{\prime}{ }_{\perp}$ negative ( -3.1). At this energy the matrix has negative dielectric constant, and so it is not transmissive. The presence of $\mathrm{LiF}$ rods with $\varepsilon^{\prime}>0$ at $25 \mathrm{vol} \%$ opens this transmission window with a hyperbolic dispersion. This region, also present in $\mathrm{KCl}-\mathrm{LiF}$, has been studied with the aid of computer simulations and experiments by M. Massaouti et al [31]. In the range from 14 to $17 \mathrm{THz}$, the same kind of dispersion exists $\left(\varepsilon^{\prime} / />0\right.$ and $\left.\varepsilon_{\perp}^{\prime}<0\right)$, so that, a slice cut parallel to the rods will channel light in a plane perpendicular to the rods [17]. In this region it is LiF that possesses negative dielectric constant. The resonance giving rise to $\varepsilon_{\perp}^{\prime}<0$ corresponds to the LiF surface modes which are excited with E perpendicular to the rods. At around $10 \mathrm{THz}, \varepsilon_{/ /}^{\prime}<0$ and $\varepsilon_{\perp}^{\prime}>0$. Consequently $\pi$-polarized light will propagate collimated along the LiF rod direction inside a transverse-cut slice of the material [17]. These three regions of indefinite permittivity are shadowed in figures $5 \mathrm{a}$ and $6 \mathrm{a}$.

As the volume fraction of $\mathrm{LiF}$ diminishes calculations show that the transmittance at $10 \mathrm{THz}$ (in perpendicular polarization) and $16 \mathrm{THz}$ (in parallel polarization) increases, while the intensity of the LiF resonances, bulk or surface, decreases. Moreover, if the reststrahlen regions of the matrix shifts to lower energies, the window at around 6.7 $\mathrm{THz}$ shifts to lower energies, broadens (both materials being transparent) and 
transmittance increases. Of course, only a part of this high transmission window will correspond to hyperbolic behavior. This corresponding to shadowed regions in Fig. 6a The rest of the window around $10 \mathrm{THz}$ (between 8 and $9.2 \mathrm{THz}$ ), matrix and rods both have positive permittivity and there is strong contrast. This might generate photonic crystal-like behavior with the appropriate microstructural size and filling fraction. For example, on approaching 9.2 THz from below, in the NaCl-LiF ( $\mathrm{r} / \mathrm{a}=0.26$ for $25 \mathrm{vol} \%$ filling fraction), $\varepsilon_{\mathrm{LiF}}^{\prime}=10.9$ while $\varepsilon^{\prime}{ }_{\mathrm{NaCl}}=0.94$, so that, according to the gap maps given in [33], a TM gap should open with $a$ around $10 \mu \mathrm{m}$ or $16.6 \mu \mathrm{m}$.

Reflectance of $\mathrm{KCl}-\mathrm{LiF}$ and $\mathrm{NaCl}-\mathrm{LiF}$ from 3 to $11 \mathrm{THz}$, reported in the literature, [14] conform to the predictions of equations 2 at small interparticle distances. In figure 7 we compare the transmittance measured in perpendicular polarization at shorter wavelengths, frequencies between 11 and $24 \mathrm{THz}$, with the expected one calculated with the Maxwell-Garnett model (eqs. 2b and 5). The samples are transverse-cut slices of $\mathrm{KCl}-\mathrm{LiF}$ grown at 6 and $2 \mathrm{~mm} / \mathrm{h}$ (rod diameters $2.6 \pm 0.2$ and $4.7 \pm 0.3 \mu \mathrm{m}$ respectively). We used $\mathrm{KCl}$ and $\mathrm{LiF}$ dielectric constant values as tabulated by Palik. We can see clearly the strong absorption that corresponds to the excitation of the surface transverse modes at the LiF rods. It shifts towards longer wavelengths (lower energies) as the LiF rod-diameter increases, as expected [21].

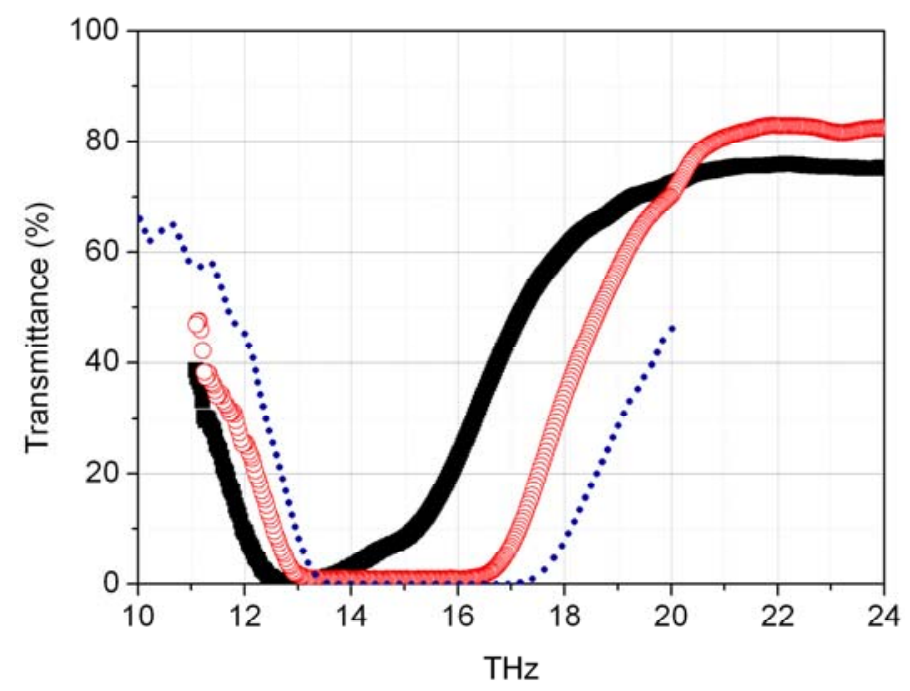

Figure 7: Transmittance of $\mathrm{KCl}-\mathrm{LiF}$ slices, $90 \mu \mathrm{m}$ thick, cut perpendicular to the rod direction (perpendicular polarization). Measurements: filled squares ( $2 \mathrm{~mm} / \mathrm{h}$ pulling rate) (black online), open circles ( $6 \mathrm{~mm} / \mathrm{h}$ pulling rate) (red online). Calculation: dotted line (blue online), using dielectric constant of components as tabulated by Palik [34]). 
In figure $5 \mathrm{~b}$ we give the calculated $\varepsilon^{\prime}$ of a lamellar eutectic, using equations 2 . In particular of the eutectic $\mathrm{CaF}_{2}-\mathrm{LiF}$ containing $42 \mathrm{vol} \% \mathrm{CaF}_{2}$. For the $\mathrm{CaF}_{2}$ and $\mathrm{LiF}$ dielectric constants between 3 and $25 \mathrm{THz}$, we used the one-mode Lorentz model with the parameters given in the figure caption. Around $15 \mathrm{THz}\left(\varepsilon^{\prime} / /=-0.95\right.$ and $\left.\varepsilon^{\prime}{ }_{\perp}=1.85\right)$ and at $18.5 \mathrm{THz}\left(\varepsilon^{\prime} / /=0.45\right.$ and $\left.\varepsilon^{\prime}{ }_{\perp}=-0.28\right)$ we have indefinite dielectric constant concomitant with a significant transmittance for a $50 \mu \mathrm{m}$ thick slab in one polarization (see figure 6b). Both regions arise at both sides of the high frequency resonance which appears with perpendicular polarization at around $16.3 \mathrm{THz}$, and so will shift and change permittivity magnitude with $\mathrm{LiF}$ volume fraction and also, beyond the long wavelength limit, with the thickness of the lamellae.

Polaritonic metamaterial predictions for these and new eutectic systems are given in figures $8 \mathrm{a}$ to $8 \mathrm{e}$. In these figures we present the real parts of the parallel and perpendicular dielectric constants and transmittances calculated for $50 \mu \mathrm{m}$ or $20 \mu \mathrm{m}$ thickness slices of the fibrous $\mathrm{NaCl}-\mathrm{LiF}, \mathrm{KCl}-\mathrm{LiF}, \mathrm{NaCl}-\mathrm{NaF}, \mathrm{CaF}_{2}-\mathrm{MgO}$ and $\mathrm{NaCl}-$ $\mathrm{LiF}-\mathrm{CaF}_{2}$ eutectics, under the long wavelength limit approximation (equations 2 and 3 ), using equation 5 . The dielectric constants were calculated with equations 3 , using as input data the ones tabulated by Palik [34]. Shadowed regions describe these frequency ranges in which dielectric constants of different sign in the two polarizations, predict hyperbolic dispersion. Real materials have, apart from the main Lorentz type resonance, other resonances of smaller magnitude due to multiphonon absorption or defects that can have strong influence on the transmittance of composites in the areas of interest

In general, in the possible regions of hyperbolic dielectric constant, the transmittance is small due to increased absorption. This is more pronounced for parallel polarization configuration. The behavior of $\mathrm{NaCl}-\mathrm{NaF}$ eutectic system is very similar to that of $\mathrm{NaCl}-\mathrm{LiF}$ (figures $7 \mathrm{a}$ and 8c), except for the increased absorption in the former due to the NaF. Note also that when the volume fraction of fibers is sufficiently small (for example $\mathrm{LiF}$ in $\mathrm{KCl}$, figure $8 \mathrm{~b}$ ), the perpendicular polarization surface resonance is not intense enough to open a hyperbolic behavior on its high energy side (surface modes excited with E perpendicular to the LiF fibers). This is even more pronounced when multiple resonances are required to describe the permittivity of the rods. 

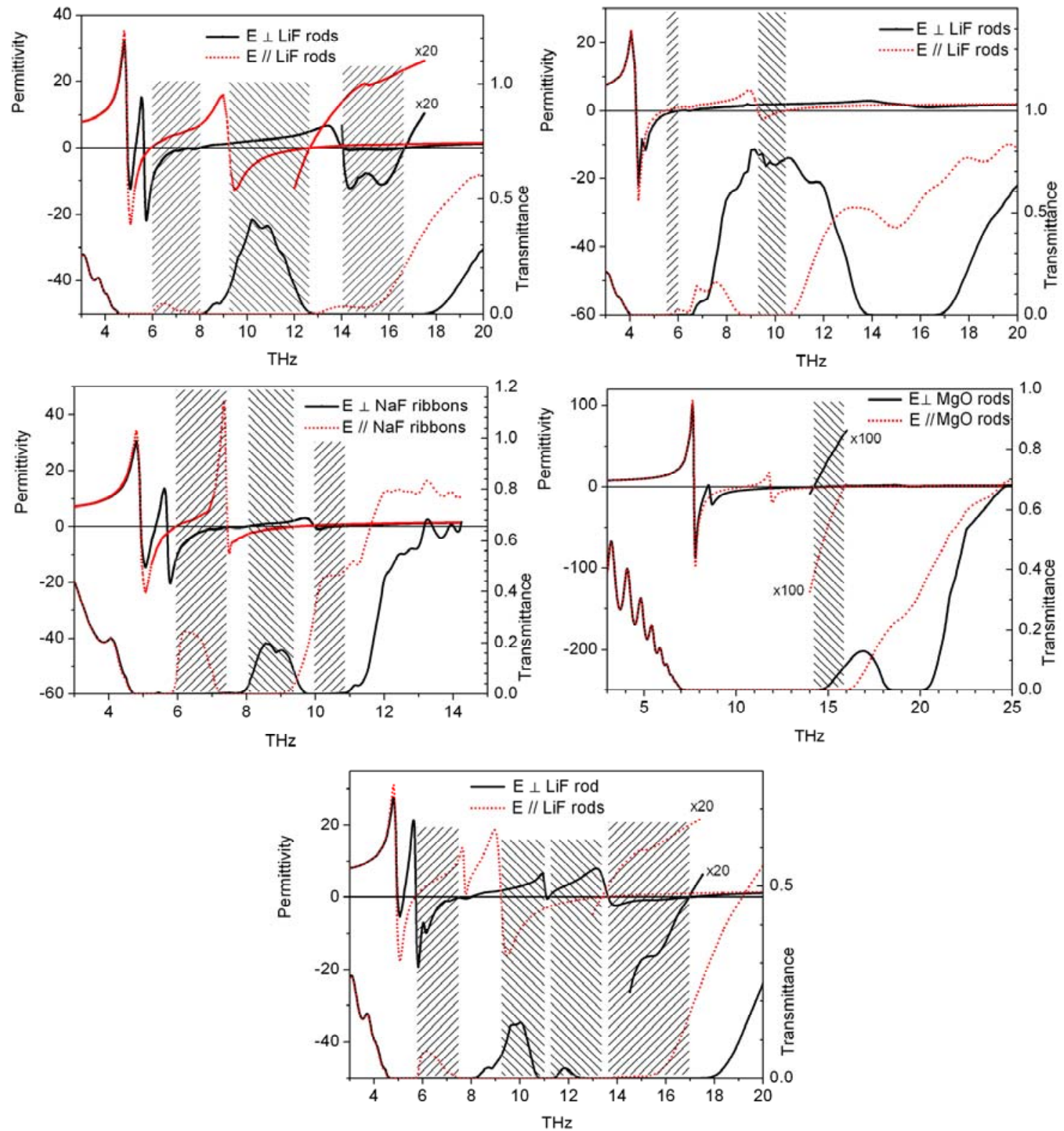

Figure 8: Calculated permittivity (real) and transmittance of slices for parallel (dotted line) and perpendicular (solid line) polarizations of the eutectics: (a) NaCl-LiF, slices $50 \mu \mathrm{m}$ thick; (b) KCl-LiF, slices $50 \mu \mathrm{m}$ thick; (c) $\mathrm{NaCl}-\mathrm{NaF}$, slices $20 \mu \mathrm{m}$ thick, (d) $\mathrm{CaF}_{2}-\mathrm{MgO}$ and (e) $\mathrm{NaCl}-\mathrm{LiF}-\mathrm{CaF}_{2}$. Calculations using the data tabulated by Palik and equations $1 \mathrm{a}$ and $1 \mathrm{~b}$. The regions where the materials have indefinite permittivity are shadowed.

For the ternary eutectic NaCl-LiF-CaF 2 , a Maxwell-Garnett type model can be employed. For parallel polarization one extra absorption due to $\mathrm{CaF}_{2}$ between the ones due to $\mathrm{NaCl}$ and $\mathrm{LiF}$ is expected (equation 2a). In the perpendicular polarization, 
surface localized modes between $\mathrm{CaF}_{2}-\mathrm{NaCl}, \mathrm{CaF}_{2}-\mathrm{LiF}$ and $\mathrm{LiF}-\mathrm{NaCl}$ would give absorptions at frequencies below the longitudinal optical modes of $\mathrm{LiF}$ and $\mathrm{CaF}_{2}$, introducing more broadened resonances around $14 \mathrm{THz}$. A behavior similar to NaCl-LiF is expected in the very small size microstructures (compare figures 8a and 8e). Apart from the $\mathrm{CaF}_{2}$ absorption, the most important difference between the ternary composite and the $\mathrm{NaCl}-\mathrm{LiF}$ is the smaller size of the microstructure and the fact that $\mathrm{LiF}$ fibers are located nearer to one another, only separated by the $\mathrm{CaF}_{2}$ phase. The spatial pattern of the interphase modes will also be affected by this microstructure, so that inter-rod localized modes might arise [16]. It is worth noticing that other ternary eutectics do exist that might substitute the $\mathrm{CaF}_{2}$ phase in the inter-LiF regions by another less absorbing phase, thus enhancing localization of light at the inter-rod space.

\section{SUMMARY}

In this work we report on several alkali halide based eutectics grown by the Bridgman method. They present highly anisotropic and homogeneous microstructures, either fibrous-like or lamellae. The ternary eutectic $\mathrm{NaCl}-\mathrm{LiF}-\mathrm{CaF}_{2}$ has also been grown, its microstructure being described here for the first time. The microstructural sizes show interparticle distances (center-to-center) from $1 \mu \mathrm{m}$ to several tens of microns.

Simple effective homogenization models have been used to calculate effective anisotropic permittivity and transmittance in the $\mathrm{THz}$ range of eutectic slices with $\mathrm{E}$ either perpendicular or parallel to the rod or lamellae composing the microstructure. This allows identifying the ranges where hyperbolic behaviour is expected together with significant transmittance. When the size of the dispersed phase particles is not very small as compared with electromagnetic wavelength, resonances are expected to shift with the size, as has been shown for the resonance around $15 \mathrm{THz}$ in KCl-LiF. Consequently, the associated hyperbolic dispersion ranges will also shift showing that the materials response can be to a certain extent tailored by the manufacture conditions. Size and shape sensitivity could also be important in real systems where some variability in the dispersed phase size and shape is expected. 
Recently published calculations of light propagation and spatial mode distributions on $\mathrm{NaCl}-\mathrm{LiF}$ and $\mathrm{KCl}-\mathrm{LiF}$ systems demonstrate that hyperbolic, indefinite permittivity causes canalization of electromagnetic waves, revealing the potentialities of this family of compounds. We have shown here that many other eutectics exists that can be chosen for a particular application. Moreover, ternary eutectics open the palette of possibilities (in properties of the components, volume fraction and microstructural sizes) at the time of choosing the appropriate material for an intended application wavelength in the $\mathrm{THz}$ range.

\section{ACKNOWLEDGEMENTS}

Authors would like to acknowledge the financial support by EU under the project NMP4-SL-2008-213669-ENSEMBLE. MFA acknowledges Ministerio de Educación, Cultura y Deporte (Spain) for the FPU scholarship. Discussions with partners of the ENSEMBLE project and especially with Maria Kafesaki (FORTH, Crete, Greece) are gratefully acknowledged. We also thank Rubén Gotor for his help in growing crystals.

\section{REFERENCES}

[1] Tonouchi M. Cutting-edge terahertz technology. Nature Photonics 2007; 1: 97-105.

[2] Cai W, Shalaev V. Optical Metamaterials: Fundamentals and Applications. New York: Springer; 2010.

[3] Tuniz A, Kuhlmey BT, Lwin R, Wang A, Anthony H, Leonhardt R, Fleming SC. Drawn metamaterials with plasmonic response at terahertz frequencies. Appl Phys Lett 2012; 9: 191101.

[4] Singh N, Tuniz A, Lwin R, Atakaramians S, Argyros A, Fleming SC, Kuhlmey BT. Fiber drawn doble split ring resonators in the terahertz range. Optical Materials Express 2012; $2: 1254-1259$.

[5] Ditinger J, Mühlig S, Rockstuhl C, Scharf T. A bottom-up approach to fabricate optical metamaterials by self-assembled metallic nanoparticles. Optical Materials Express 2012; 2: 269-278.

[6] Huang KC, Povinelli ML, Joannopoulos JD. Negative effective permeability in polaritonic photonic crystals. Appl Phys Lett 2004; 85: 543-545.

[7] Yao J, Liu Z, Liu Y, Wang Y, Sun CH, Bartal G, Stacy AM, Zhang X. Optical Negative Refraction in Bulk Metamaterials of Nanowires. Science 2008; 321: 930.

[8] Fang A, Koschny T, Soukoulis CM. Optical anisotropic metamaterials: negative refraction and focusing. Phys Rev B 2009; 79: 245127.

[9] Pawlak DA, Kolodziejak K, Turczynski S, Kisielewski J, Rozniatowski K, Diduszko R, Kaczkan M and Malinowski M. Self-Organized, rodlike, Micrometer-Scale 
Microstructure of $\mathrm{Tb}_{3} \mathrm{Sc}_{2} \mathrm{Al}_{3} \mathrm{O}_{12}-\mathrm{TbScO}_{3}: \operatorname{Pr}$ Eutectic. Chem. Mater. 2006; 18:24502457.

[10] Pawlak DA. Metamaterials and Photonic Crystals - Potential Applications for SelfOrganized Eutectic Micro- and Nanostructures. Scientia Plena 2008;4-014801.

[11] Orera VM, Pena JI. Directional Solidification. In: Bansal NP, Boccaccini AR, editors. Ceramics and Composites Processing Methods. Hoboken (New Jersey): The American Ceramic Society, published by John Wiley \& Sons, Inc; 2012, p 417-457.

[12] Pawlak DA, Turczynski S, Gajc M, Kolodziejak K, Diduszko R, Rozniatowski K, Smalc J, Vendik I. How Far Are We from Making Metamaterials by Self-Organization? The Microstructure of Highly Anisotropic Particles with an SRR-Like Geometry. Adv Functional Materials 2010; 20:1116-1124.

[13] Llorca J, Orera VM. Directionally solidified eutectic ceramic oxides. Prog Mater Sci 2006; 51: 711-809.

[14] Reyes-Coronado A, Acosta MF, Merino RI, Orera VM, Kenanakis G, Katsarakis $\mathrm{N}$, et al Selforganization approach for $\mathrm{THz}$ polaritonic metamaterials. Optics Express. 2012; 20(13):14663-82.

[15] Foteinopoulou S, Kafesaki M, Economou E, Soukoulis C. Two-dimensional polaritonic photonic crystals as terahertz uniaxial metamaterials. Phys. Rev. B. 2011; $84: 35128$.

[16] Myroshniychenko V, Stefanski A, Manjavacas A, Kafesaki M, Merino RI, Orera VM, Pawlak DA García de Abajo FJ. Interacting plasmon and phonon polaritons in aligned nano- and microwires. Optics Express 2012; 20:10879-10887.

[17] Basharin AA, Kafesaki M, Economou EN, Soukoulis CM. Superlensing effects in anisotropic eutectic metamaterials in the THz range. AIP Conf. Proc. 2011; 1398: 64.

[18] Basharin AA, Kafesaki M, Economou EN, Soukoulis CM. Backward wave radiation from negative permittivity waveguides and its use for $\mathrm{THz}$ subwavelength imaging. Optics Express 2012; 20: 12752-12760.

[19] Tropf WJ, Thomas ME, Harris TJ. Properties of Crystals and Glasses. In: Handbook of Optics: Devices, Measurements and Properties. Eds. M. Bass, second edition. New York: Mc. Graw Hill Inc. 1995, p 33.3 - 33.101.

[20] Torquato S. Random Heterogeneous Materials: Microstructure and Macroscopic Properties. New York: Springer-Verlag Inc. (2002).

[21] Ruppin R, Englman R. Optical phonons of small crystals. Rep Prog Phys 1970; 33: 149.

[22] Penfold C, Hellawell A. Microstructure of Alkali Halide Eutectics LiF-NaF and NaF-NaCl. J. Am. Ceram. Soc. 1965; 48: 133-135.

[23] Cooksey DJS, Munson D, Wilkinson MP, Hellawell A. The freezing of some continuous binary eutectic mixtures. Phil. Mag. 1964; 10: 745-769.

[24] Larrea A, Contreras L, Merino R.I., Llorca J, Orera V.M. Microstructure and physical properties of $\mathrm{CaF}_{2}-\mathrm{MgO}$ eutectics produced by the Bridgman method. J. Mater. Res. 2000;15:1314-1319.

[25] Chouiyakh S, Gimeno F, Pena JI, Contreras L, Orera VM. Thermoluminescence properties of $\mathrm{CaF}_{2}-\mathrm{LiF}: \mathrm{Mn}$ eutectic melt grown composites. Phys Chem News 2003; 13 : 139-143.

[26] Acosta MF, Merino RI, Orera VM. Crystal Growth and Microstructure of KCl-LiF directionally solidified eutectics. $5^{\text {th }}$ International Workshop on Crystal Growth Technology, June 2011, Berlin.

[27] Larrea A, Orera V. NaCl-assisted growth of micrometer-wide long single crystalline fluoride fibres. Opt Mater 2005; 27, 1726-29. 2005;27:1726-1729. 
[28] Larrea A, Orera V. Porous crystal structures obtained from directionally solidified eutectic precursors. J Cryst Growth 2007;300:387-393.

[29] Oliete PB, Pena JI, Larrea A, Orera VM, LLorca J, Pastor JY, Martín A, and Segurado J. Ultra-High-Strength Nanofibrillar $\mathrm{Al}_{2} \mathrm{O}_{3}-\mathrm{YAG}-\mathrm{YSZ}$ Eutectics. Adv Mater 2007; 19: 2313-2318.

[30] Orera VM, Larrea A, Merino RI, Rebolledo MA, Valles JA, Gotor R, Pena JI. Novel photonic materials made from ionic eutectic compounds. Acta Physica Slovaca 2005; 55: 261-269.

[31] Massaouti M, Basharin AA, Kafesaki M, Acosta MF, Merino RI, Orera VM, Economou EN, Soukoulis CM, Tzortzakis S. Eutectic epsilon-near-zero metamaterial terahertz waveguides. Optics Letters 2013; 38: 1140-1142.

[32] Proix F, Racek R, Balkanski M. Study of Interface Phonons in Lamellar Eutectics. J. Vac. Sci. Technol 1973;10: 663- 664.

[33] Joannopoulos JD, Johnson SG, Winn JN, Meade RD. Photonic Crystas: Molding the flow of Light. 2nd ed. Princeton, New Jersey. Princeton University Press; 2008.

[34] Handbook of Optical Constants of Solids (vol. III). Ed. Palik ED. London: Academic Press; 1998. 
Table 1: Some eutectics grown in our lab.

\begin{tabular}{|c|c|c|c|c|c|}
\hline $\begin{array}{c}\text { Eutectic } \\
\text { A - B (- C) }\end{array}$ & $\begin{array}{l}\text { Micros- } \\
\text { tructure }\end{array}$ & $\mathrm{f}(\mathrm{vol} \%)$ & $\begin{array}{c}\text { Phonon } \\
\text { modes A } \\
(\mathrm{THz}) \\
v_{\mathrm{TA}}-v_{\mathrm{LA}}\end{array}$ & $\begin{array}{c}\text { Phonon } \\
\text { modes B } \\
(\mathrm{THz}) \\
v_{\mathrm{TB}}-v_{\mathrm{LB}}\end{array}$ & Notes \\
\hline \multicolumn{6}{|l|}{ Binary PED } \\
\hline KCl-LiF & $\mathrm{F}$ & $7 \% \mathrm{LiF}$ & $4.26-6.41$ & $9.17-19.75$ & $\begin{array}{l}{[26,} \\
31] \\
\end{array}$ \\
\hline $\mathrm{NaCl}-\mathrm{LiF}$ & $\mathrm{F}$ & $25 \% \mathrm{LiF}$ & $4.92-7.91$ & $9.17-19.75$ & {$[27]$} \\
\hline NaCl-NaF & $\mathrm{R}$ & $25 \% \mathrm{NaF}$ & $4.92-7.91$ & $7.31-12.35$ & $\begin{array}{c}\text { This } \\
\text { work; } \\
{[22,} \\
23]\end{array}$ \\
\hline $\mathrm{CaF}_{2}-\mathrm{MgO}$ & $\mathrm{F}$ & $9 \% \mathrm{MgO}$ & $7.73-14.18$ & $12.05-21.52$ & {$[24]$} \\
\hline $\mathrm{CaF}_{2}-\mathrm{LiF}$ & $\mathrm{L}$ & $42 \% \mathrm{CaF}_{2}$ & $7.73-14.18$ & $9.17-19.75$ & {$[25]$} \\
\hline $\mathrm{BaF}_{2}-\mathrm{NaF}$ & $\mathrm{L}$ & $43 \% \mathrm{NaF}$ & $5.52-9.56$ & $7.31-12.53$ & {$[28]$} \\
\hline \multicolumn{6}{|l|}{ Ternary PED } \\
\hline $\begin{array}{c}\mathrm{NaCl}- \\
\mathrm{LiF}-\mathrm{CaF}_{2}\end{array}$ & $\mathrm{~F}$ to $\mathrm{L}$ & $\begin{array}{l}6 \% \mathrm{CaF}_{2} \\
30 \% \mathrm{LiF}\end{array}$ & $4.92-7.91$ & $\begin{array}{l}7.73-14.18 \\
9.17-19.75\end{array}$ & $\begin{array}{l}\text { This } \\
\text { work }\end{array}$ \\
\hline
\end{tabular}

F: Fibers; L: Lamellae; R: Ribbons 